\title{
Shen Shuai II Recipe attenuates apoptosis in 5/6th renal ablation/infarction rats by inhibiting p53 and mitochondrial pathway of apoptosis
}

\author{
Meng Wang \\ Shuguang Hospital \\ Jing Yang \\ Shuguang Hospital \\ Liuyi Yang \\ Shuguang Hospital
}

Chen Wang ( $\square$ chenwang42@126.com )

Shuguang Hospital Affiliated to Shanghai University of Traditional Chinese Medicine https://orcid.org/0000-0002-6444-8184

\section{Research}

Keywords: Shen Shuai $₫$ Recipe, Chronic kidney disease, Apoptosis, P53, Mitochondria

Posted Date: November 11th, 2019

DOI: https://doi.org/10.21203/rs.2.17133/v1

License: (9) This work is licensed under a Creative Commons Attribution 4.0 International License. Read Full License 


\section{Abstract}

Background Chronic kidney disease (CKD) has become a serious challenge to global public health. Apoptosis is closely related to the evolution of CKD. In China, it has been noted that Chinese herbal medicine may be suitable for the treatment of CKD. Shen Shuai II Recipe (SSR) is a classic formula for the treatment of CKD in the clinic and proves the renprotective effects. However, the underlying mechanism remains unclear. The main purpose of this study was to investigate whether SSR could reduce apoptosis in $5 / 6$ renal ablation/infarction (A/I) hypoxia model by regulating p53 and mitochondrial pathway of apoptosis.

Methods 28 days after the $5 / 6(A / I)$ surgery, Sprague-Dawley rats were randomly divided into four groups: sham group, 5/6 (A/I) group, 5/6 (A/I) + SSR group and 5/6 (A/I) +Losartan group (5/6 (A/I) +LOR). After 56 days of treatment, we mainly assessed the translocation of apoptotic factors in mitochondrial apoptosis pathway, the degree of mitochondrial dysfunction and the nuclear and mitochondrial translocation of p53. Furthermore, we detected the interaction of p53 with anti-apoptotic Bcl-xL and Bcl-2 proteins.

Results SSR significantly inhibited the mitochondrial accumulation of pro-apoptotic protein Bax and Puma and release of cytochrome $c$ from mitochondria to cytosol in the $5 / 6(\mathrm{~A} / \mathrm{I})$ model. In addition, SSR improved the mitochondrial function and inhibited the nuclear and mitochondrial translocation of p53. SSR suppressed the p53 transactivation and the interaction of p53 with Bcl-xL and Bcl-2.

Conclusions These results suggested that SSR could exert anti-apoptotic effects in the 5/6 (A/I) hypoxia model by inhibiting p53 transcriptional dependent and independent pro-apoptotic functions and the mitochondrial pathway of apoptosis.

\section{Background}

Chronic kidney disease (CKD) has considerably increased worldwide and remains a major global health problem[1]. Different types of CKD ultimately progress to the end-stage renal disease (ESRD) through the same pathological process of renal fibrosis, resulting in a decline in quality of life and a substantial economic burden[2]. Therefore, finding cost-effective treatment of CKD is a key challenge for public health.

Apoptosis is a mechanism of cell self-destruction involved in a variety of biological events[3]. Despite its role in physiology, abnormal apoptosis is implicated in various diseases related to ischemia, autoimmunity, and viral infections, and contributes to pathological progression in the course of acute and chronic renal injury[4,5]. In mammalian cells, mitochondrial pathway of apoptosis, so-called intrinsic pathway, plays a crucial role in activating apoptosis. Mitochondria are not only the site where Bcl-2 family members interact, but also the origin of signals that initiate the activation of caspases through release of apoptotic factors, such as cytochrome c[3]. P53, previously described as a tumor suppressor, is now considered to be a key component of the mitochondrial apoptosis pathway under cell stress. In response 
to various stimuli, such as hypoxia, DNA damage and reactive oxygen species (ROS), p53 induces cell death through dual action, including activation of target genes and transcriptional-independent signal mechanism[6].

Recently, it has been noted that Chinese herbal medicine may be suitable for the treatment of CKD, because the efficacy and safety of long-term treatment is very important in the management of this lifethreatening disease. Shen Shuai $\triangle$ Recipe (SSR), a classic formula for the treatment of CKD in the clinic, has been widely used for more than 20 years and become the effective prescription for the treatment of patients with CKD. Although our previous studies have demonstrated that SSR could improve renal injury and fibrosis in 5/6 renal ablation/infarction (A/I) model, the underlying mechanism under the efficacy remained elusive[7]. In addition, using renal ultrasound detection and blood gas analysis, we confirmed that the renal blood flow decreased and the oxygen consumption increased in 5/6 (A/I) model[8,9]. More importantly, we preliminarily studied that SSR could reduce apoptosis, which may be interconnected with the improvement of renal blood flow and oxygen consumption[8]. In this study, we aimed to elucidate whether SSR could exert anti-apoptotic effects by P53 and mitochondrial pathway of apoptosis.

\section{Methods}

Animals and drugs

Eight-week-old male Sprague-Dawley (SD) rats, weighing approximately $190 \mathrm{~g}-210 \mathrm{~g}$, were purchased from SLAC Laboratory Animal Co., Ltd. (Shanghai, China). The rats had free access to food and water and maintained at $23 \pm 3^{\circ} \mathrm{C}$, relative humidity of $55 \pm 15 \%$ with a $12 \mathrm{~h}$ light $/ 12 \mathrm{~h}$ dark cycle. SSR consists of Salvia miltiorrhiza bge $15 \mathrm{~g}$, Codonopsis pilosula $15 \mathrm{~g}$, Angelica sinensis $15 \mathrm{~g}$, Rheum palmatum $15 \mathrm{~g}$, Folium perillae $15 \mathrm{~g}$, Epimedium $15 \mathrm{~g}$, Peach kernel $15 \mathrm{~g}$, Ligusticum chuanxiong $15 \mathrm{~g}$ and Coptis chinensis $6 \mathrm{~g}$. The preparation of SSR was consistent with the previous standard protocol and the gavage dose $(10 \mathrm{~mL} / \mathrm{kg}$, containing $6 \mathrm{~g} / \mathrm{mL}$ of the original drug) in this study was determined on the basis of the previous studies[7,8]. Losartan tablets $(100 \mathrm{mg} /$ tablet) were purchased from MSD Pharmaceutical Co., Ltd.(Hangzhou, China). Losartan was dissolved and diluted by saline with a concentration of $5 \mathrm{mg} / \mathrm{mL}$ of solution.

Rat 5/6 (A/I) model and animal study protocol

The 5/6 (A/I) hypoxia model was established as described in our previous work[7-10]. Four weeks after 5/6 (A/I) operation, 30 rats were randomized into three groups: 5/6 (A/I) group, 5/6 (A/I) + SSR group (treated with $10 \mathrm{~mL} / \mathrm{kg}$ of SSR daily by gavage, $\mathrm{n}=10)$ and $5 / 6(A / \mathrm{I})+$ Losartan group $(5 / 6(A / I)+L O R$, treated with $6 \mathrm{~mL} / \mathrm{kg}$ of losartan daily by gavage, $n=10)$. This study also included a group of 10 shamoperated rats. After 8 weeks of administration, the rats were anaesthetized with sodium pentobarbital (40 $\mathrm{mg} / \mathrm{kg}$, i.p.) and the kidney tissues were collected for molecular detection.

Isolation of cytosolic and mitochondrial fractions 
Using tissue mitochondria isolation kit (Inventbiotech, Beijing, China), the cytosolic and mitochondrial fractions were extracted at $4^{\circ} \mathrm{C}$ by centrifugal column filter and multiple centrifugation method. About $30 \mathrm{mg}$ of tissue was placed in a filter cartridge. The tissue was ground with a plastic rod for one minute by pushing the tissue against the surface of the filter repeatedly. The filter cartridge was centrifuged at $16,000 \mathrm{~g}$ for 30 seconds. The pellet was resuspend by vortexing briefly. The homogenates were first centrifuged at $700 \mathrm{~g}$ for one minute to remove cell debris and nuclei and then centrifuged at $16,000 \mathrm{~g}$ for 10 min to collect the supernatant as cytosolic fraction. The pellet was resuspend in $200 \mu \mathrm{l}$ buffer $B$ and then centrifuged at $8,000 \mathrm{~g}$ for $5 \mathrm{~min}$. The supernatant was further centrifuged at $16,000 \mathrm{~g}$ for $30 \mathrm{~min}$ to collect the pellet as mitochondrial fraction.

Isolation of cytosolic and nuclear fractions

The cytosolic and nuclear fractions were extracted at $4{ }^{\circ} \mathrm{C}$ by the multiple centrifugation method, using nuclear and cytoplasmic protein extraction kit (Beyotime, Shanghai, China). About $50 \mathrm{mg}$ of tissue was cut into pieces and homogenized gently in the cytoplasmic protein extraction reagent with a glass tissue grinder. The homogenates were centrifuged at $1500 \mathrm{~g}$ for $5 \mathrm{~min}$ to collect the supernatant as cytosolic fraction. The pellet was resuspend in $50 \mu \mathrm{l}$ nuclear protein extraction reagent and then centrifuged at $16,000 \mathrm{~g}$ for $10 \mathrm{~min}$ to collect the supernatant as nuclear fraction.

Caspase 3 activity analysis

The caspase 3 activity was detected by caspase 3 activity assay kit (Jiancheng Bioengineering Institute, Nanjing, China). About $50 \mathrm{mg}$ of tissue was ground in lysis buffer with a glass homogenizer and then centrifuged at $12,000 \mathrm{~g}$ for $15 \mathrm{~min}$. The supernatant protein was collected and quantified by Bradford method. The supernatant containing $200 \mu$ g protein was incubated with $50 \mu \mathrm{L}$ reaction buffer and $5 \mu \mathrm{L}$ caspase- 3 substrate at $37^{\circ} \mathrm{C}$ for $4 \mathrm{~h}$. The caspase 3 activity was measured at absorbance of $405 \mathrm{~nm}$.

Immunoprecipitation analysis

Immunoprecipitation analysis was performed as previously described[7]. Briefly, Kidney tissues were lysed on ice for $15 \mathrm{~min}$ in lysis buffer. Approximately $300 \mu \mathrm{g}$ of total protein was incubated overnight at 4 ${ }^{\circ} \mathrm{C}$ with anti-P53 (Santa Cruz, USA) followed by precipitation with $70 \mu$ l of protein A/G-Plus-Agarose (Santa Cruz, USA) for $4 \mathrm{~h}$ at $4{ }^{\circ} \mathrm{C}$. Non-specific IgG (Proteintech) was used as the control. The precipitated complexes were washed in immunoprecipitation buffer and then, resuspended in $30 \mu \mathrm{l}$ of $2 \times$ loading buffer and boiled for 5 min.

Hoechst 33,342 assay

The Hoechst 33,342 staining (Beyotime, Shanghai, China) was used to identify the morphology of apoptotic nuclei. The three-micrometer-thick sections were deparaffinized and then exposed to the Hoechst 33,342 solution for $5 \mathrm{~min}$ at $37^{\circ} \mathrm{C}$ in the dark. The positive nuclei were observed by fluorescence microscope (Nikon Eclipse80i, Japan) at 200x magnification. 
Western blot

Immunoblotting detection was performed as previously described[7,8]. In the present study, the primary antibodies used were anti-cytochrome c (1:1000, Abcam, UK), anti-Bcl2 (1:500, Genspan, USA), anti-Bax (1:1000, Abcam, UK), anti-SDHB (1:1000,Abcam, UK), anti-parp (1:1000,CST,USA), anti-Puma (1:500, Santa Cruz, USA), anti-ATPB (1:1000, Proteintech, USA), anti-caspase9 (1:1000, Abcam,UK) ,anti-P53 (1:1000,CST,USA), anti-P-p53(1:1000, Abcam, UK) and anti-Bcl-xL (1:1000,Abcam, UK). Lamin B1 (1:1000, Abclonal, China), VDAC1 (1:1000, Abcam,UK) and Gapdh(1:2000, Proteintech, USA) were used as the controls. The antigens on the blots were visualized by using the enhanced chemiluminescence kit (Thermo Fisher Scientific, USA).

Real-time PCR

Total RNA was extracted from kidney tissue using Trizol reagent (Beyotime, Shanghai, China) and reverse transcribed to cDNA using a TaqMan RNA Reverse Transcription Kit (Takara, Dalian, China) according to manufacturer's instructions. Then, qPCR was performed on an ABI StepOnePlus Real-Time PCR System (Applied Biosystems, Foster City, USA) with SYBR Green Master Mix (Yeasen, Shanghai, China) according to the instructions provided with the kit. Relative expression was determined by the $2^{-\Delta \Delta C T} \operatorname{method}$. $\beta$ actin was used as an internal control. The PCR reaction conditions were as follows: $95^{\circ} \mathrm{Cfor} 5 \mathrm{~min}$, followed by 40 cycles of 10 s at $95^{\circ} \mathrm{C}$ and 30 s at $60{ }^{\circ} \mathrm{C}$. Primer sequences for Puma were: forward ACTGCCAGCCTTGCTTGTC and reverse AGTCCTTCAGCCCTCCCTTC; Bax primer sequences were: forward GGCGATGAACTGGACAAC and reverse CCGAAGTAGGAAAGGAGG; Noxa primer sequences were: forward GTTACCGCCTGAATTCGCAG and reverse AGTTATGTCCGGTGCACTCC; $\beta$-actin primer sequences were: forward GAGAGGGAAATCGTGCGT and reverse GGAGGAAGAGGATGCGG.

Statistical analysis

All data were presented as mean \pm SEM and analyzed by one-way analysis of variance with LSD-t's multiple comparison, using SPSS software (version 18.0, SPSS Inc., Chicago, USA). P $<0.05$ was considered statistically significant.

\section{Results}

SSR exerted anti-apoptotic effects in the 5/6 (A/I) hypoxia model

As a key executor of apoptosis, caspase-3 is implicated in the proteolysis of many critical proteins[11]. In this study, we first detected the level of caspase-3 activity. Compared with sham group, 5/6 (A/I) operation markedly increased the caspase-3 activity(Fig. 1a). Conversely, SSR significantly attenuated the increase in active caspase-3 production compared with 5/6 (A/I) group. Upon caspase activation, cleavage of parp by caspases has been identified as one of the first biochemical markers of apoptosis[12]. Therefore, the level of parp cleavage was detected by immunoblotting. As shown in Fig. 1(b) and (c), SSR significantly inhibited the cleavage of parp, which was consistent with the result of 
caspase-3 activity test. The mitochondrial pathway of apoptosis requires activation of caspase-9, which then activate caspase-3[13]. In this study, immunoblotting analysis showed that SSR also inhibited the level of cleaved caspase-9 in the 5/6 (A/I) model (Fig. 1b and c). Using the TUNEL assay, our previous studies reported that SSR treatment for 8 weeks dramatically reduced the number of apoptotic cells in the $5 / 6(A / I)$ hypoxia model[8]. In the present study, we further analysed the morphology of apoptotic nuclei by Hoechst 33,342 staining. As shown in Fig. 1(d), the normal nuclei were uniformly stained without nuclear condensation or fragmentation and the apoptotic cells showed the abnormal nuclear size and nuclear fragmentation or condensation. The morphology of nuclear abnormalities induced by $5 / 6(A / I)$ operation was obviously ameliorated by SSR treatment.

SSR inhibited the mitochondrial accumulation of pro-apoptotic Bax and Puma proteins and release of cytochrome c from mitochondria to cytosol in the $5 / 6(A / I)$ model

The mitochondrial pathway of apoptosis is mainly triggered by Bax oligomerization in the mitochondria and subsequent release of apoptogenic factors from the mitochondrial intermembrane space, such as cytochrome c[14]. Therefore, in the present study, we enriched the mitochondrial fraction from the remnant kidneys and analyzed the translocation of Bax and cytochrome $\mathrm{c}$ proteins by immunoblotting. In the sham group, cytochrome $c$ was mainly located in the mitochondria and the 5/6 (A/I) operation significantly increased the translocation of cytochrome $c$ to the cytosolic fraction (Fig. 2a and b). Furthermore, compared with the sham group, 5/6 (A/I) hypoxia model displayed an higher oligomerization of Bax in the mitochondria (Fig. 2c and d). Conversely, SSR for 8 weeks treatment markedly inhibited the accumulation of Bax in the mitochondira and the release of cytochrome $c$ into the cytosol. In addition, we detected the level of p53 upregulated modulator of apoptosis (Puma), a proapoptotic BH3-only protein that was shown to localize to mitochondria and activate Bax[14], in the mitochondrial and cytosolic samples. Our results demonstrated that the accumulation of Puma in mitochondria showed the same pattern as that of Bax in each group (Fig. $2 \mathrm{c}$ and d).

Furthermore, mitochondrial dysfunction is a well-known pathogenic factor in ischemic renal injury and the destruction of mitochondrial integrity is more sensitive to the insertion of Bax[15,16]. Therefore, we detected the levels of mitochondrial respiratory chain-associated proteins. Compared with the sham group, the SDHB and ATPB, the subunits of complex- $₫$ and complex-区[17], significantly down-regulated in $5 / 6$ renal (A/I) model group, which was dramatically reversed by SSR (Fig. 2e and f).

SSR suppressed the nuclear translocation and transactivation of p53 in the 5/6 (A/I) model

Since p53 is the most critical molecule upstream of Puma and Bax and that the nuclear translocation of p53 represents its activation[18], we extracted the nuclei from the remnant kidney tissue to analyze the protein level of p53 in the nuclear fraction. Our results showed that the protein levels of p53 and its phosphorylation were significantly up-regulated in the nuclear fraction relative to control subjects (Fig. 3a and $b$ ). Furthermore, qRT-PCR results demonstrated that the mRNA expression of Puma, Bax and Noxa, the targets directly by p53 driven, was markedly up-regulated in the 5/6 (A/I) model (Fig. 3c). However, SSR significantly inhibited the expression of p53 target proapoptotic genes and the translocation of p53 
from the cytosol to the nucleus in 5/6 (A/I) rats, suggesting that SSR could regulate transcriptiondependent apoptotic activity of p53.

SSR inhibited the mitochondrial translocation and activation of p53 in the 5/6 (A/I) model

In addition to the transcriptional-dependent pro-apoptotic effect of p53, previous studies have confirmed that in different cell types, the mitochondrial translocation of p53 leads to the interaction of p53 with Bcl$x \mathrm{~L}$ and $\mathrm{Bcl}-2$, and inhibits their anti-apoptotic effects[6,19-21]. In this study, we found that the $5 / 6(A / I)$ operation increased p53 and its phosphorylation in the mitochondria, which was inhibited by SSR treatment for 8 weeks (Fig. $4 a$ and b). To assess the interaction of p53 with anti-apoptotic Bcl-xL and Bcl2 proteins, p53 was immunoprecipitated and the degree of interaction was analyzed by immunoblotting. As shown in Fig. 4(c) and (d), co-immunoprecipitation and immunoblotting studies demonstrated a direct interaction p53 with Bcl-xL and Bcl-2 in the mitochondria. Quantitative analysis showed that the binding of p53-Bcl2 or p53-Bcl-xL was increased in model group(Fig. 4e). On the contrary, SSR significantly inhibited the interaction of $\mathrm{p} 53$ with $\mathrm{Bcl}-\mathrm{xL}$ and $\mathrm{Bcl}-2$.

\section{Discussion}

In this study, we demonstrated that SSR showed the anti-apoptotic effects in the 5/6 (A/I) hypoxia model by mitochondrial pathway. SSR inhibited the accumulation of Bax and Puma in the mitochondria and release of cytochrome $c$ into the cytosol, which ultimately led to the less active caspase 3 and lower nuclear condensation or fragmentation. In addition, SSR inhibited the nuclear translocation and transcriptional activity of p53 in CRF rats, which was reflected in the decrease of Puma, Bax and Noxa mRNA expression. More importantly, using co-immunoprecipitation and immunoblotting analysis, we found that SSR not only significantly reduced translocation of P53 to mitochondria, but also inhibited the direct interaction between P53 and antiapoptotic Bcl-2 or Bcl-xL protein.

Previous studies demonstrated that in different disease models, inhibition of apoptosis showed significant renal protective effect. Du et al[22]. reported that the activation of vitamin D receptor protected against lipopolysaccharide (LPS)-induced acute kidney injury by blocking renal tubular epithelial cell apoptosis. Huang et al[23]. demonstrated that L3MBTL2, a novel polycomb group protein, improved renal injury by the p53 apoptosis pathway in renal tubular cells. Peng et al[16]. reported that p53 and mitochondrial pathway of apoptosis were involved in the susceptibility of diabetic models to ischemic acute kidney injury. These studies prompted us to further study the possible role of apoptosis in the $5 / 6$ (A/I) hypoxia model, as well as the mechanisms of SSR attenuating renal injury and fibrosis. The mitochondria, as the central organelle of the intrinsic apoptotic pathway, is regulated by pro- and antiapoptotic Bcl-2 family members[24]. Upon stress, the integrity of outer mitochondrial membrane (OMM) is impaired, which is sensitized to the oligomerization and insertion of Bax and subsequently lead to the release of cytochrome $c$ into the cytosol[25,26]. Our previous studies have reported that $5 / 6(A / I)$ operation could trigger mitochondrial pathway of apoptosis by increasing accumulation of Bax in the mitochondria and release of cytochrome $c$ into the cytosol[10]. In this study, we enriched mitochondria 
from the remnant kidneys. Our results showed that SSR markedly inhibited the intrinsic apoptotic pathway, improved the pathological morphology of nucleus, and lowered caspase 3 activation, which could be associated with renal protective effect of SSR. In addition, as the main oxygen consumption organelle, the mitochondria are uniquely dependent on the continuous availability of oxygen for the function of the electron transport chain[27]. During hypoxia, the dysfunction and dynamic disruption of mitochondria attract Bax attack and insertion and ensuing apoptosis[28]. Therefore, we detected the level of mitochondrial electron transport chain proteins. SSR significantly increased the protein levels of complexe- $\mathbb{Z}$ and complexe- $\mathrm{V}$ subunits, which could be helpful to maintain mitochondrial integrity, resist Bax insertion and cytochrome c release.

Previous studies have reported that persistent hypoxia could be the main trigger of p53 activation in the process of renal injury and p53-mediated induction of Puma and Bax was responsible for apoptosis in ischemic kidneys[29-32]. However, it is important that, in the process of mitochondrial apoptosis pathway, the suborganellar localization of p53 affects its function. In the nucleus, transcriptional activation of p53 up-regulates the expression of Puma and Bax involved in mitochondrial apoptosis pathway[33]. Therefore, we isolated the nuclear and cytoplasmic components from the remnant kidney tissues to detect the translocation of p53 to the nucleus. Our results showed that the levels of P53 and its phosphorylation in the nuclear fraction were dramatically elevated in 5/6 (A/I) model. SSR significantly suppressed the nuclear translocation and transactivation of p53, evideced by the down-regulation of Bax, Puma and Noxa mRNA expression. More importantly, in response to stress, cytoplasmic p53 is directly involved in the mitochondrial pathway of apoptosis by translocation to mitochondria. P53 in mitochondria can directly bind to the anti-apoptotic members Bcl-xL and Bcl-2 and neutralize their inhibitory effects on proapoptotic Bax to stimulate mitochondrial outer membrane permeabilization (MOMP) and subsequent apoptosis. In this study, we found that SSR inhibited the accumulation and phosphorylation of p53 in the mitochondria. Furthermore, using the co-immunoprecipitation analysis, we showed that $5 / 6(A / I)$ operation increased the binding of p53 to bcl2 or bcl-xl in the mitochondria, which was reversed by SSR.

\section{Conclusions}

Taken together, we demonstrated that SSR could attenuate apoptosis in the $5 / 6(\mathrm{~A} / \mathrm{I})$ hypoxia model by the mitochondrial pathway of apoptosis. Furthermore, activation of p53-induced target genes and transcriptional-independent apoptotic activity of p53 could amplify the intrinsic pathway of apoptosis, which was inhibited by SSR treatment for 8 weeks. However, the relationship between p53 transcriptional dependent and independent pro-apoptotic functions and the regulatory effect of SSR on them need to be further clarified in future study.

\section{Abbreviations}

CKD:Chronic kidney disease; SSR:Shen Shuai खRecipe; 5/6 (A/I): 5/6 ablation/ infarction; SD:SpragueDawley; ESRD:End-stage renal disease; ROS:Reactive oxygen species; Puma:P53 upregulated modulator 
of apoptosis; LPS:Lipopolysaccharide; OMM:Outer mitochondrial membrane; MOMP:Mitochondrial outer membrane permeabilization

\section{Declarations}

\section{Acknowledgements}

Not applicable.

\section{Authors' contributions}

CW designed the study and participated in data analysis and interpretation. MW, JY and LYY finalized the experimental work, interpreted the results and prepared Figs. MW wrote the paper, CW and JY edited and revised. All authors read and approved the final version of manuscript.

\section{Funding}

This study was supported by the National Natural Science Foundation of China General Projects (81573946, 81973770), Shanghai Three Year Project of Traditional Chinese Medicine (ZY(2018-2020)FWTX-7005). The funding bodies did not participate in the design of the study and collection, analysis, and interpretation of data and writing of the manuscript.

\section{Availability of data and materials}

The datasets used and/or analyzed during the current study are available from the corresponding author on reasonable request.

\section{Ethics approval and consent to participate}

The animal procedures were approved by the Animal Experiment Ethics Committee of Shanghai University of Traditional Chinese Medicine in accordance with the principles outlined in the NIH Guide for the Care and Use of Laboratory Animals.

\section{Consent for publication}

Not applicable.

\section{Competing interests}

The authors declare that they have no competing interests.

\section{References}

1. Feehally J. Chronic kidney disease: Health burden of kidney disease recognized 
by UN. Nat Rev Nephrol. 2011; 8:12-13.

2. Chen KC, Peng CC, Hsieh CL, Peng R. Exercise ameliorates renal cell apoptosis in chronic kidney disease by intervening in the intrinsic and the extrinsic apoptotic pathways in a rat model. Evid Based Complement Alternat Med. 2013:368450.

3. Jeong SY, Seol DW. The role of mitochondria in apoptosis. BMB Rep. 2008; 41:

$11-22$.

4. Saikumar P, Dong Z, Mikhailov V, Denton M, Weinberg JM, Venkatachalam MA. Apoptosis: definition, mechanisms, and relevance to disease. Am J Med. 1999; 107: 489-506.

5. Sanz AB, Santamaría B, Ruiz-Ortega M, Egido J, Ortiz A. Mechanisms of renal apoptosis in health and disease. J Am Soc Nephrol. 2008; 19:1634-1642.

6. Sansome C, Zaika A, Marchenko ND, Moll UM. Hypoxia death stimulus induces translocation of p53 protein to mitochondria. Detection by immunofluorescence on whole cells. FEBS Lett. $2001 ; 488: 110-115$.

7. Wang M, Yang LY, Yang J, Wang Chen. Shen Shuai IIRecipe attenuates renal injury and fibrosis in chronic kidney disease by regulating NLRP3 inflammasome and Sirt1/Smad3 deacetylation pathway. BMC Complement Altern Med. 2019;

19:107-115.

8. Wang M, Yang J, Zhou Y, Wang C. ShenShuai II Recipe attenuates apoptosis and renal fibrosis in chronic kidney disease by increasing renal blood flow and improving oxygen consumption. Evid Based Complement Alternat Med. 2018: 7602962.

9. Deng AH, Tang T, Singh P, Wang C, Satriano J, Thomson SC, Blantz RC. Regulation of oxygen utilization by angiotensin II in chronic kidney disease. Kidney Int. 2009; 75: 197-204.

10. Wang M, Yang LY, Yang J, Zhou Y, Wang C. Magnesium lithospermate B attenuates renal injury in 5/6 renal ablation/infarction rats by mitochondrial pathway of apoptosis. Biomed Pharmacother. 2019; 118:109316-109323. 
11. Fernandes-Alnemri T, Litwack G, Alnemri ES. CPP32, a novel human apoptotic

protein with homology to Caenorhabditis elegans cell death protein Ced-3 and mammalian interleukin-1 beta-converting enzyme. J Biol Chem. 1994; 269:30761-30764.

12. Xu G, Li S, Liu X, Gao P, Chen X, Wang H, Zhang M, Yang Y, Gao GF, Zhang F.

PARP-1 mediated cell death is directly activated by ZIKV infection. Virology. 2019; 537: 254-262.

13. Fadeel B, Orrenius S. Apoptosis: a basic biological phenomenon with

wide-ranging implications in human disease. J Intern Med. 2005; 258:479-517.

14. Bock FJ, Tait Stephen WG. Mitochondria as multifaceted regulators of cell death.

Nat Rev Mol Cell Biol. 2019:1-16.

15. Ryu DR, Yu MR, Kong KH, Kim H, Kwon SH, Jeon JS, Han DC, Noh H.

Sirt1-hypoxia-inducible factor-1a interaction is a key mediator of tubulointerstitial damage in the aged kidney. Aging Cell. 2019; 18:12904.

16. Peng J, Li X, Zhang D, Chen J, Su Y, Smith SB, Dong Z. Hyperglycemia, p53, and

mitochondrial pathway of apoptosis are involved in the susceptibility of diabetic models to ischemic acute kidney injury. Kidney Int. 2015; 87:137-150.

17. Xiao Y, Zhou Y, Lu Y, Zhou K, Cai W. PHB2 interacts with LC3 and SQSTM1 is required for bile acids-induced mitophagy in cholestatic liver. Cell Death Dis. 2018; 9: 160-170.

18. Li X, Yu Q, Chen FS, Tan WF, Zhang ZL, Ma H. Inhibiting aberrant p53-PUMA feedback loop activation attenuates ischaemia reperfusion-induced neuroapoptosis and neuroinflammation in rats by downregulating caspase 3 and the NF-KB cytokine pathway. $\mathrm{J}$ Neuroinflammation. 2018; 15:250-262.

19. Marchenko ND, Zaika A, Moll UM. Death signal-induced localization of p53

protein to mitochondria. A potential role in apoptotic signaling. J Biol Chem. 2000; 275:16202-16212.

20. Arima Y, Nitta M, Kuninaka SJ, Zhang DW, Fujiwara T, Taya Y, Nakao M, Saya H.

Transcriptional blockade induces p53-dependent apoptosis associated with translocation of p53 to mitochondria. J Biol Chem. 2005; 280:19166-19176.

21. Moll UM, Marchenko N, Zhang XK. p53 and Nur77/TR3 - transcription factors 
that directly target mitochondria for cell death induction. Oncogene. 2006; 25: 4725-4743.

22. Du J, Jiang S, Hu Z, Tang S, Sun Y, He J, Li Z, Yi B, Wang J, Zhang H, Li YC. Vitamin D

receptor activation protects against lipopolysaccharide-induced acute kidney injury through suppression of tubular cell apoptosis. Am J Physiol Renal Physiol. 2019; 316: 1068-1077.

23. Huang H, Xu C, Wang Y, Meng C, Liu W, Zhao Y, Huang XR, You W, Feng B, Zheng

ZH, Huang Y, Lan HY, Qin J, Xia Y. Lethal (3) malignant brain tumor-like 2 (L3MBTL2) protein protects against kidney injury by inhibiting the DNA damage-p53-apoptosis pathway in renal tubular cells. Kidney Int. 2018; 93:855-870.

24. Kelekar A, Thompson CB. Bcl-2-family proteins: the role of the $\mathrm{BH} 3$ domain in apoptosis. Trends Cell Biol. 1998;8:324-330.

25. Beere HM, Wolf BB, Cain K, Mosser DD, Mahboubi A, Kuwana T, Tailor P,

Morimoto RI, Cohen GM, Green DR. Heat-shock protein 70 inhibits apoptosis by preventing recruitment of procaspase-9 to the Apaf-1 apoptosome. Nat Cell Biol. 2000; 2:469-475.

26. Harris $\mathrm{MH}$, Thompson $\mathrm{CB}$. The role of the Bcl-2 family in the regulation of outer mitochondrial membrane permeability. Cell Death Differ. 2000; 7:1182-1191.

27. Thomas LW, Ashcroft M. Exploring the molecular interface between hypoxia-inducible factor signalling and mitochondria. Cell Mol Life Sci. 2019; 76: 1759-1777.

28. Brooks C, Cho SG, Wang CY, Yang T, Dong Z. Fragmented mitochondria are sensitized to Bax insertion and activation during apoptosis. Am J Physiol Cell Physiol. 2011; 300:447455 .

29. Tang C, Ma Z, Zhu J, Liu Z, Liu Y, Liu Y, Cai J, Dong Z. P53 in kidney injury and repair: Mechanism and therapeutic potentials. Pharmacol Ther. 2019; 195:5-12.

30. Sermeus A, Michiels C. Reciprocal influence of the p53 and the hypoxic pathways.Cell Death Dis. 2011; 2:164-174.

31. Singaravelu K, Padanilam BJ. p53 target Siva regulates apoptosis in ischemic kidneys. Am J Physiol Renal Physiol. 2011; 300:1130-1141. 
32. Zhang D, Liu Y, Wei Q, Huo Y, Liu K, Liu F, Dong Z. Tubular p53 regulates multiple genes to mediate AKI. J Am Soc Nephrol. 2014; 25:2278-2289.

33. Kruiswijk F, Labuschagne CF, Vousden KH. p53 in survival, death and metabolic health: a lifeguard with a licence to kill. Nat Rev Mol Cell Biol. 2015; 16:393-405.

Figures 


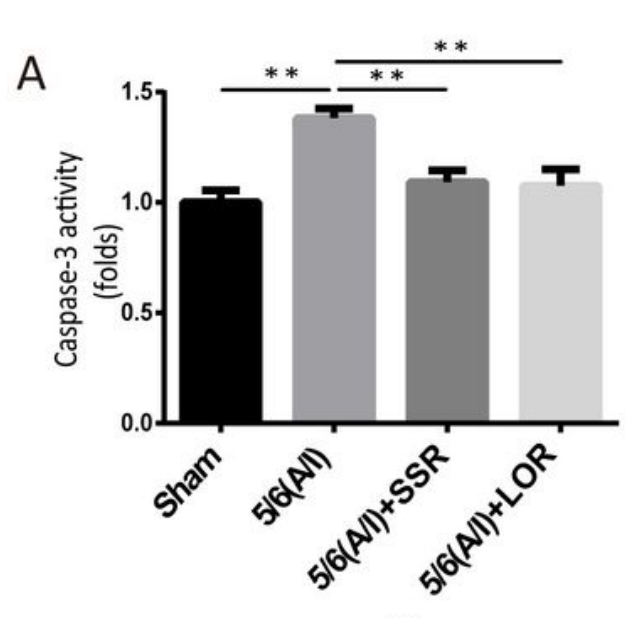

B
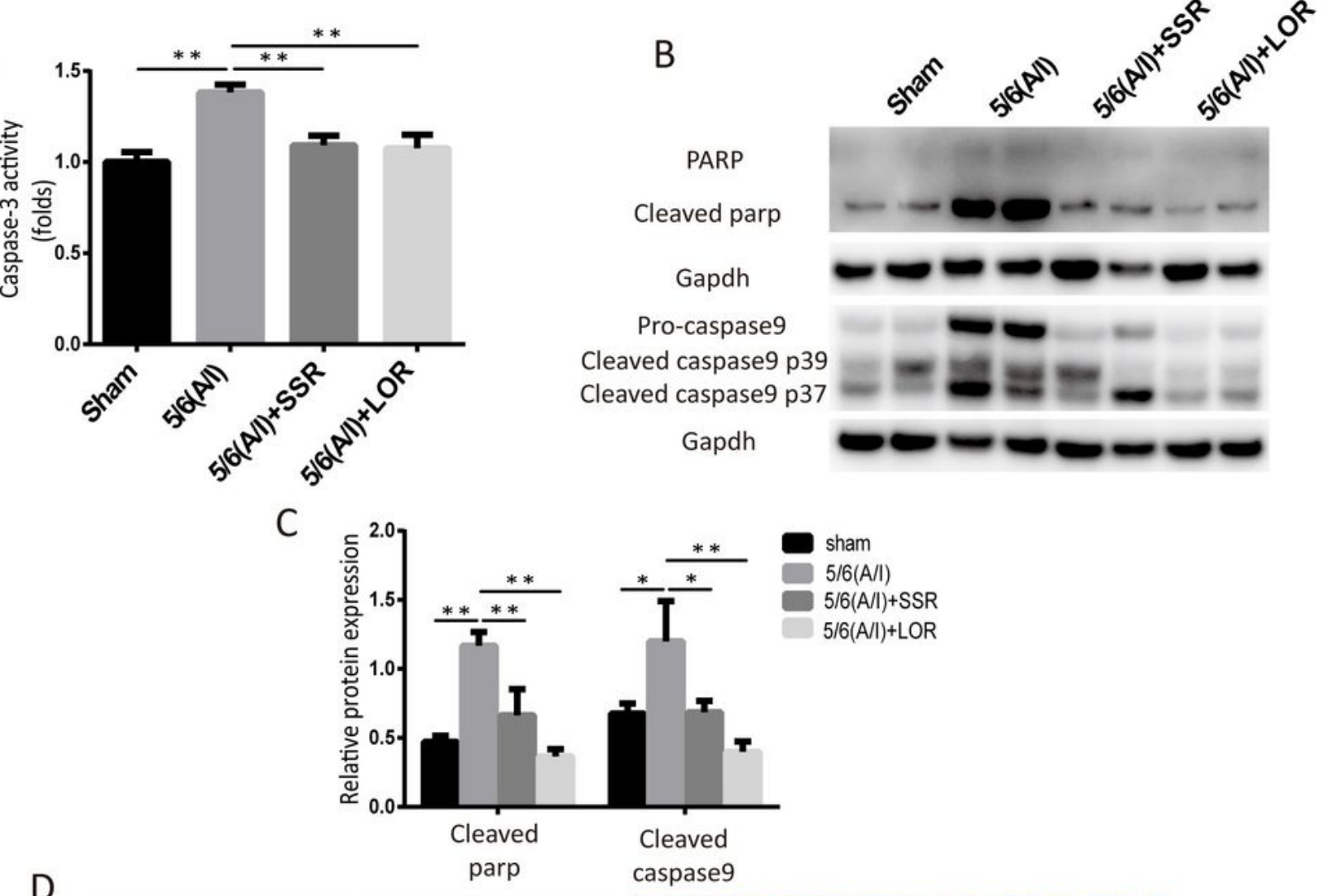

D
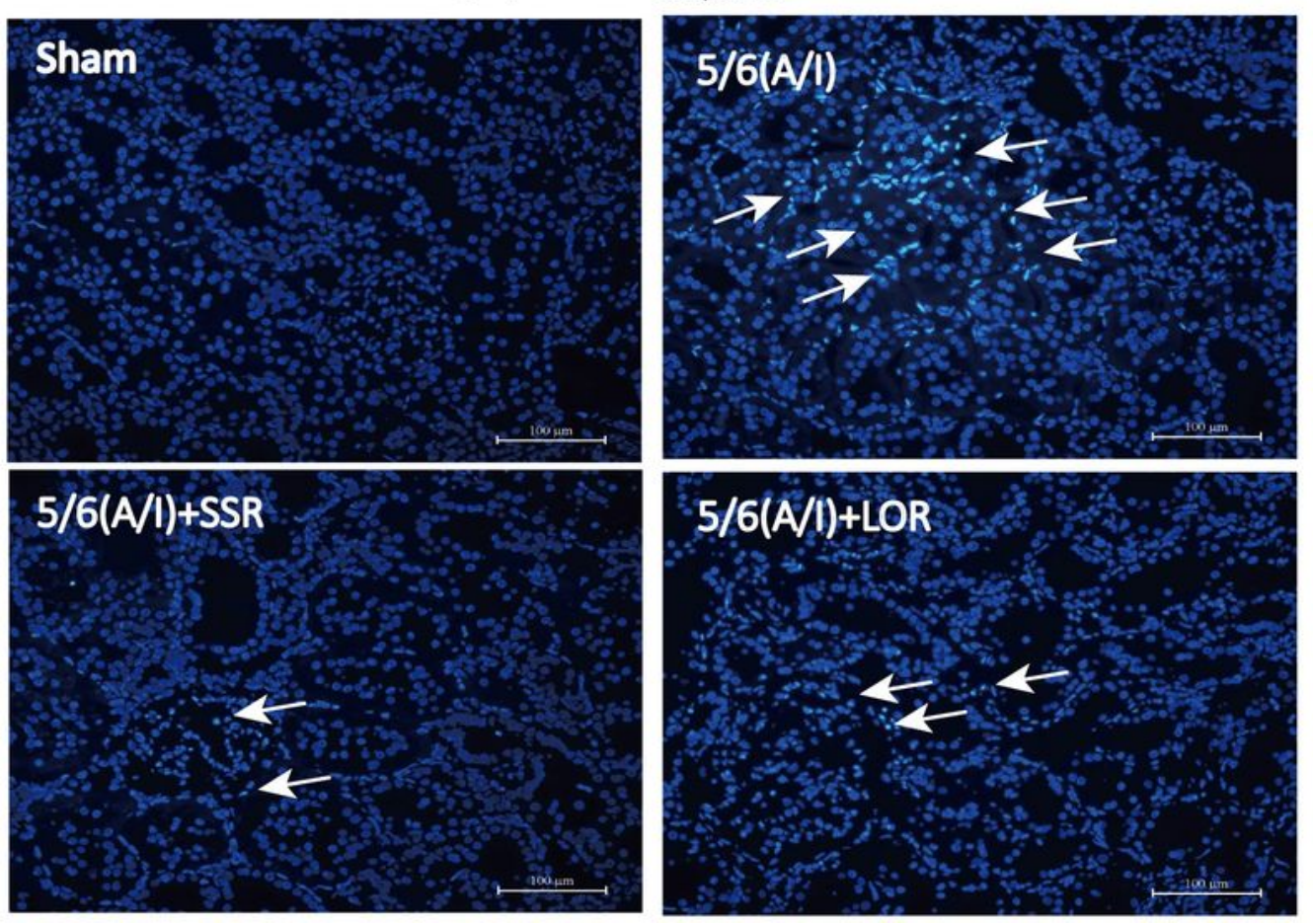

Figure 1

SSR exerted anti-apoptotic effects in the 5/6 (A/I) hypoxia model. (a) The remnant kidney tissues were collected to detect the caspase-3 activity by activity test kit. $(n=4)(b)$ The protein levels of cleaved parp and cleaved caspase- 9 were measured by immunoblotting. (c) Western blot quantification of cleaved parp and cleaved caspase-9 levels. $(n=4)(d)$ Representative micrographs of nuclear morphology were determined by Hoechst 33342 staining. $200 \times$ magnification Values are mean $\pm S E M .{ }^{*} P<0.05,{ }^{* *} P<0.01$. 


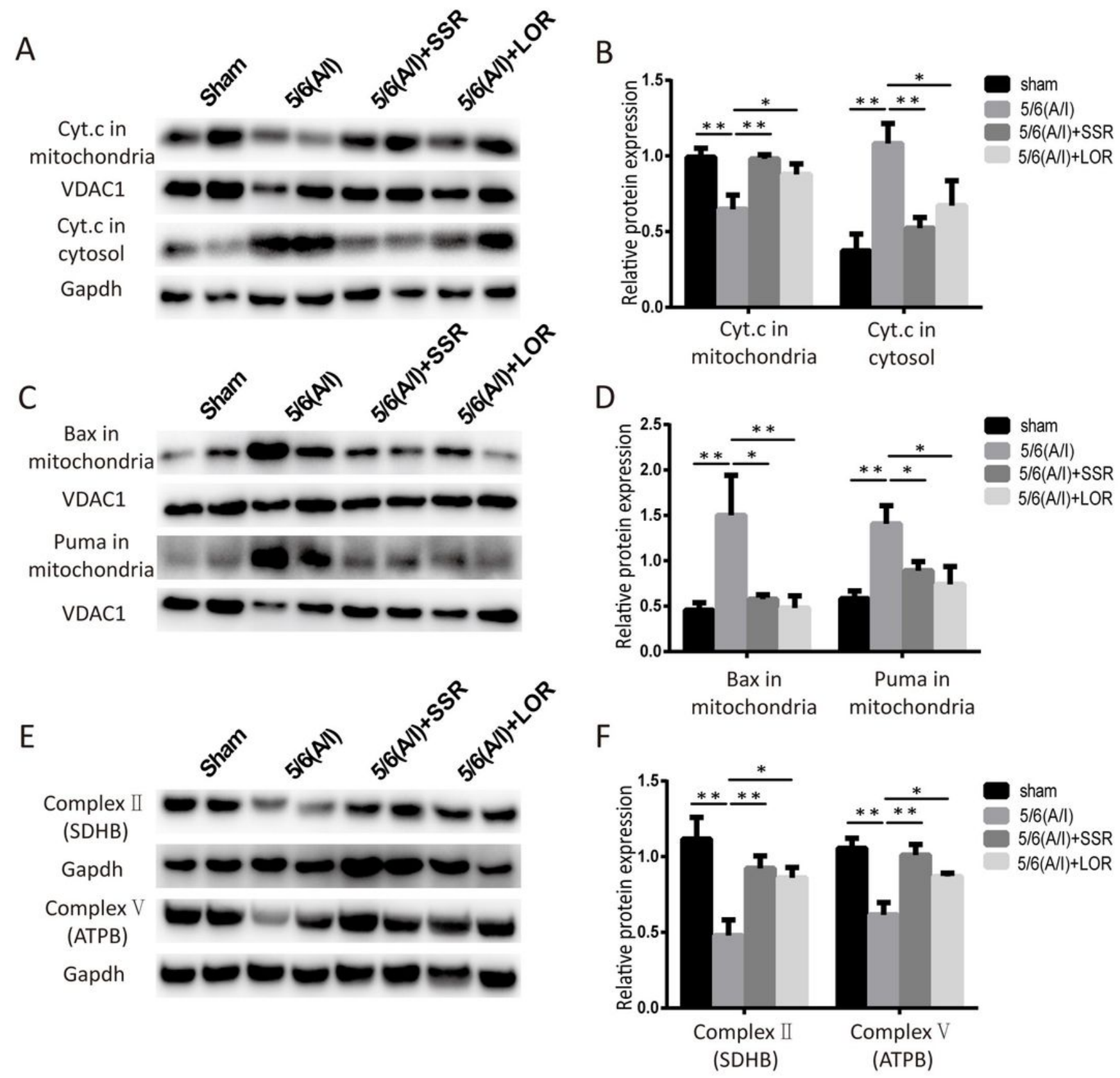

Figure 2

SSR inhibited the oligomerization of pro-apoptotic protein Bax and Puma in the mitochondria and release of cytochrome c into the cytosol in the 5/6 (A/I) model. (a) Immunoblotting analysed the protein levels of mitochondrial and cytosolic cytochrome c (Cyt. c). Gapdh and VDAC1 were used as loading controls. (b) Quantification of the relative protein levels of Cyt.c in the mitochondria and cytosol. $(n=4)(c)$ Immunoblotting analysed the protein levels of mitochondrial Bax and Puma. (d) Western blot

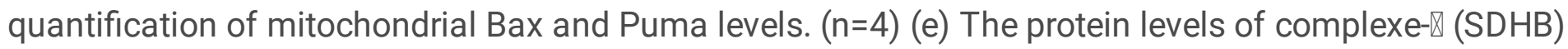
and complexe-V (ATPB) subunits were determined by immunoblotting. (f) Western blot quantification of SDHB and ATPB levels. $(n=4)$ Values are mean \pm SEM. ${ }^{*} P<0.05, * * P<0.01$. 

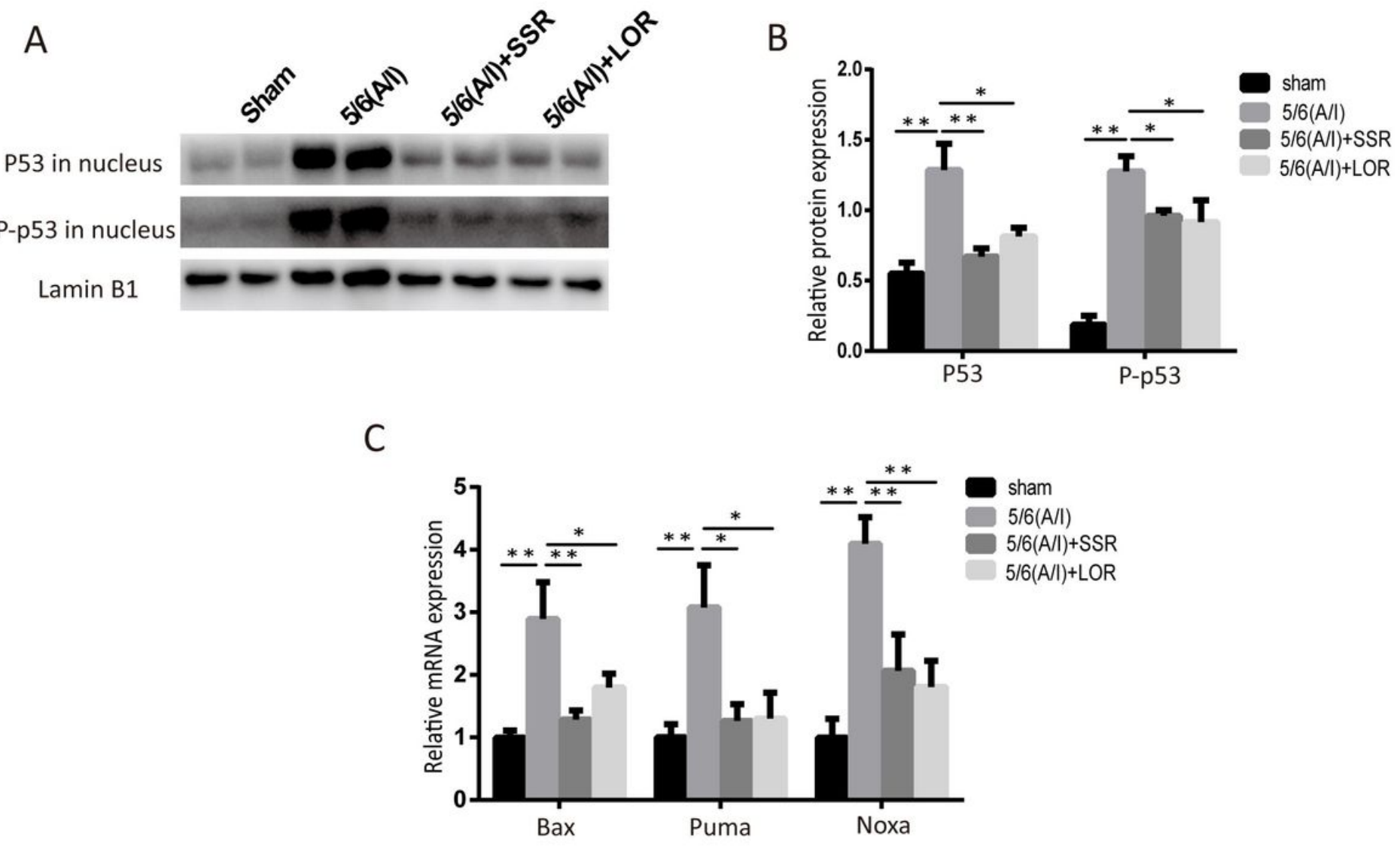

\section{Figure 3}

SSR suppressed the nuclear translocation and transactivation of p53 in the 5/6 (A/I) model. (a) Immunoblotting analysed the protein levels of nuclear P53 and its phosphorylation (P-p53). (b) The ratio of P53 and P-p53 to Lamin B1 protein was calculated. (n=4) (c) The levels of Bax, Puma and Noxa mRNA expression were determined by real-time PCR. The abundance of each mRNA was normalized to $\beta$-actin and data were expressed as fold increase over control. $(n=4)$ Values are mean $\pm S E M .{ }^{*} P<0.05, * \star P<0.01$. 

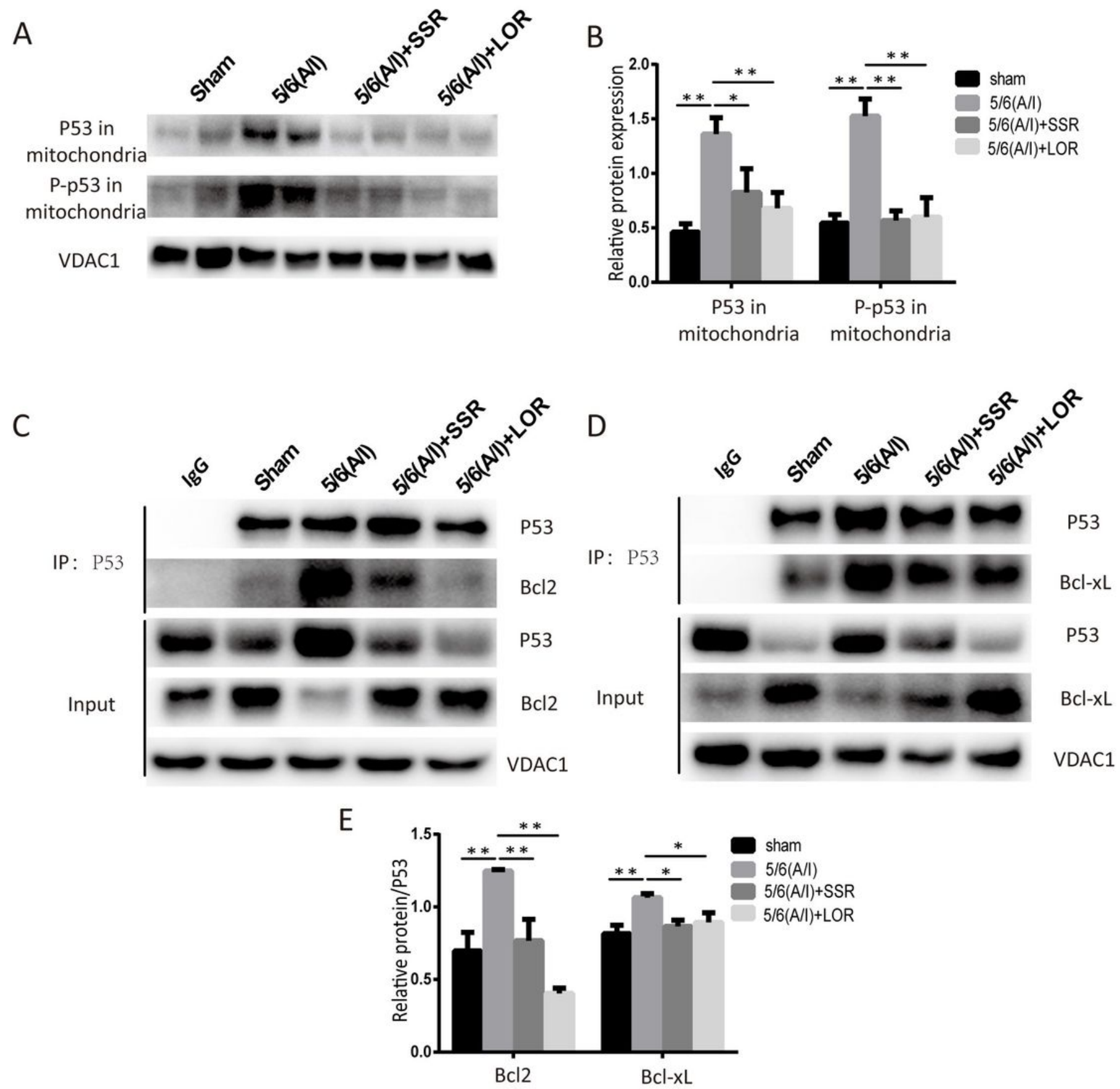

Figure 4

SSR inhibited the mitochondrial translocation and activation of p53 in the $5 / 6$ (A/I) model. (a) The protein levels of the mitochondrial P53 and P-p53 were analysed by immunoblotting. (b) Quantification of the relative protein levels of p53 and P-p53 in the mitochondria. $(n=4)(c$ d) Co-immunoprecipitation and western blot analysed the interaction $\mathrm{p} 53$ with $\mathrm{Bcl}-\mathrm{xL}$ and $\mathrm{Bcl}-2$ proteins in each group. (e) The ratio of $B c l-x L$ and Bcl-2 to precipitated P53 protein was calculated. $(n=3)$ Values are mean $\pm S E M$. ${ }^{*} P<0.05$, $\star * P<0.01$.

\section{Supplementary Files}


This is a list of supplementary files associated with this preprint. Click to download.

- originalwesternblots.ppt 